\title{
Adaptive High-Performance Method for Numerical Simulation of Unsteady Complex Flows with Number of Strong and Weak Discontinuities
}

\author{
Alexander Vinogradov ${ }^{1}$, Vladimir Volkov ${ }^{1}$, Vladimir Gidaspov ${ }^{1}$, \\ Alexander Muslaev ${ }^{1}$, and Peter Rozovski \\ ${ }^{1}$ Moscow State Aviation Institute, Volokolamskoe shosse, 4, Moscow, RUSSIA 125871 \\ 2 Trapezo, 37 Natoma Street, San Francisco, CA 94105 USA \\ peter@trapezo.com
}

\begin{abstract}
Here we discuss results of further development of numerical method for computer simulation of unsteady flows with number of strong and weak discontinuities in case of nonequilibrium homogeneous condensation. This method is based on idea of explicit tracking of discontinuity surfaces (shock waves, rarefaction waves, contact surfaces) during calculation process. The results for generation of silver clusters in dense vapors during unsteady spherical expansion closed vacuum chamber are presented.
\end{abstract}

\section{Introduction}

The solution of many real problems requires numerical treatment of unsteady flows with nonequilibrium processes. Flow field can include strong (shock waves and contact surfaces) and weak (boundary characteristics of expansion waves) discontinuities. As result of interaction of discontinuities their number changes with time. Currently two classes of numerical methods are used for treatment of such flows.

First class consists of shock-capturing methods. They use the same algorithm for all grid points without special procedures for calculation the points on discontinuity surfaces and their main advantage is uniformity. It results in their easy coding. But even best types of them (TVD, ENO, etc.) have disadvantage of spreading discontinuity to 2-3 grid cells. In case of contact surface such spreading is even worse due to unphysical growing of its length with time. It is possible to avoid this problem by using small grid cells near contact surface or special adaptive grids. But it violates the uniformity of algorithm.

Second class of methods includes the methods of tracking all or most intensive discontinuities. Their main principles have been formulated few decades ago and their effectiveness was proved many times. But due to common opinion that discontinuity tracking is extremely complex and poorly formalized process the number of investiga- 
tions carried out by above methods is very small. In most of them only limited constant number of strong discontinuities is tracked.

The advantage of methods with explicit tracking of discontinuities is simple calculation of hybrid grids with steady regular sub-grid and adaptive moving sub-grid. The last relates to tracked discontinuities and moving boundaries. It results in highaccuracy of calculations even in case of small number of nonmoving grid points.

In our previous publications [1,2] we proposed the numerical technique, which combines the advantages of both classes of methods: uniformity of algorithm and automatic explicit tracking of existed initially and generated later strong/weak discontinuities. This technique was successfully used for simulation of non-reacting gas expansion flow in vacuum chamber with solid [1] and porous [2] walls. Here we present the generalization of this technique for reacting flows with nonequilibrium homogeneous condensation.

\section{Mathematical Model of Unsteady Flows of Condensing Vapors}

The model is based on quasi-one-dimensional unsteady mass, momentum and energy transfer conservation laws written in differential form in areas of continuity solution and integral form at discontinuity surfaces. Flow is considered as inviscid and with no heat-conduction.

The corresponding system of equations for binary mixture of inert gas and condensing vapor has the next form:

$$
\frac{\partial}{\partial t}\left[\begin{array}{c}
\rho F \\
\rho u F \\
\rho e_{0} F \\
\rho \gamma_{j} F
\end{array}\right]+\frac{\partial}{\partial r}\left[\begin{array}{c}
\rho u F \\
\left(\rho u^{2}+p\right) F \\
\rho u\left(e_{0}+p \rho^{-1}\right) F \\
\rho u \gamma_{j} F
\end{array}\right]=\left[\begin{array}{c}
0 \\
p \frac{d F}{d r} \\
0 \\
\rho W_{j} F
\end{array}\right]
$$

Where $\mathrm{t}$ is time, $\mathrm{r}$ is linear coordinate, $e_{0}=e+u^{2} / 2, \rho, u, p, e$ are density, velocity, pressure and internal energy of mixture, $F=r^{v}, v=0,1,2$ for plane, cylindrical and spherical symmetry, $\gamma_{j}$ are mole-mass concentrations of clusters with $\mathrm{j}$ atoms, $W_{j}$ are rates of concentration change as a result of nonequilibrium homogeneous condensation. In last equation index $\mathrm{j}$ varies from 1 to $\infty$. The special transformation is used to approximate infinite system of kinetic equations in (1) by finite system of $\mathrm{N}$ equations [3].

The system (1) is completed by state equations 


$$
e=\gamma_{A} e_{A}(T)+\sum_{j=1}^{N} \gamma_{j} e_{j}(T), \rho=\frac{p}{R T}\left[\gamma_{A}+\sum_{j=1}^{N} \gamma_{j}\right]^{-1}
$$

And condition of constant number of atoms

$$
\gamma_{A}=\text { const }, \sum_{j=1}^{N} j \gamma_{j}=\text { const }
$$

Where $\mathrm{T}$ is temperature, $\gamma_{A}$ is mole-mass concentration of inert gas, $\mathrm{R}$ is universal gas constant.

The formulae and methods for calculation $W_{j}, e_{A}(T), e_{j}(T)$ are presented in [3], there also we discuss the methods of selection the appropriate value for $\mathrm{N}$.

\section{Numerical Method}

The suggested numerical method is based on the next principles:

- the flow is calculated by grid-characteristics method by $\mathrm{t}=$ const layers,

- the whole flow region is divided to sub-regions with continuity parameters in them, and their boundaries are surfaces of strong/weak discontinuities or external left/right boundaries of the region,

- $\quad$ numerical grid consists from non-moving and moving points. The last ones are points of discontinuities. Pairs of values corresponding left and right limits of continuum solution describe parameters in them. If discontinuity is stable (it has the same type on the next time step), the left/right values satisfy to integral relations for this type of discontinuity,

- for crossing points of two strong discontinuities the left/right values don't satisfy to such relation. The formation of new discontinuity surfaces occurs and determination of number, types and parameters of these discontinuities should be obtained from solution of corresponding Riemann problem;

- the standard time step is selected from the Courant condition. But if crossing of any discontinuities happens before next time, the exact time of nearest crossing is taken;

- the calculation process is divided into gas-dynamic half-step (calculation of $\mathrm{u}, \mathrm{p}, \mathrm{T}$ from calculated previously concentrations and right-hand-sides of kinetic equations) and kinetic half-step (calculation of concentrations by integration of kinetic equations);

- the possibility of generation of shock wave in initially continuous sub-region is analyzed. The coordinate and time of such generation point is calculated from crossing point of characteristics of the same type;

- flow parameters in each of grid nodes are calculated from characteristic conditions. The base points can locate as on previous time layer as on discontinuities surfaces. That means that all base points relates to one sub-region of 
continuum solution. The resulting system of equations is solved numerically and if there is no convergence for given number of iterations, the time step is decreased.

Any grid points relates to one of the following types: symmetry axis, solid wall, shock wave, contact surface, discontinuity characteristic, usual characteristic, trajectory and nonmoving usual grid point. From physical consideration it is clear that 18 types of crossing can take place.

For interactions shock wave/shock wave or shock wave/contact surface the structure and parameters of resulting flow are calculated from generalized solution of Riemann problem on case of reacting gases with variable ratios of specific heats. It can be shown that for thermodynamic model of inert gas/condensing vapor mixture [3] this problem has unique solution.

After solution of Riemann problem initial parameters of all generated discontinuities are known. For extra-intensive expansion waves the several additional usual characteristics are put into wave. Their number is proportional to pressure gradient in expansion wave. All generated discontinuities have the same coordinate but different parameters.

For shock wave/solid wall or shock wave/symmetry axis the parameters of reflected shock are calculated from Rankine-Hugoniot type conditions for multicomponent gas and condition $\mathrm{u}=0$ behind the shock.

In case of flow with cylindrical or spherical symmetry near symmetry axis $(r=0)$ the intensity of shock wave is infinite. The point of shock wave/symmetry axis interaction is special point with infinite values of shock velocity and pressure behind shock. The flow in such case can be calculated from known solution of Huderlay-LandauStanukovich by special algorithm.

The interactions of usual characteristics with usual characteristic, discontinuity characteristic, contact surface, solid wall, shock wave, symmetry axis and discontinuity characteristic with contact surface, shock wave, discontinuity characteristic, solid wall, symmetry axis are limiting cases of above situations. Further tracking of given characteristic or its exchange to characteristic of another family at interaction with solid wall or contact surface is determined from flow properties.

Parameters in points of interactions of trajectories with usual or discontinuity characteristics are calculated by usual method of characteristics. For trajectory/solid wall interaction parameters on trajectory are taken from point behind shock.

The main advantage of proposed method is avoiding need of a priory knowledge of flow structure. As it can be seen from presented far results, in some cases it is principally impossible due to extra-complexity of flow. The formal character of algorithm which consists from standard steps of solution of standards problems allow one to calculate flow region uniformly without changing program logic for new class of flows. The researcher needs only to know the coordinates, types and parameters of discontinuities on initial time layer. So the suggested method has all advantages of "shock-capturing" numerical schemes but allow obtain exact flow picture.

Of course as in any numerical scheme there are some "fitting" parameters like number of usual characteristics in extensive expansion waves, which should be known before calculation. Also in some cases the number of tracking discontinuities begins to grow seriously. That results in computer memory leak. In real calculations the special 
"destruction" procedure for tracked weak (strong) discontinuities with small difference between left and right values of gradients (parameters) is applied. Their "destruction" condition is the error of integral conservation laws.

After determination of parameters of discontinuities on the next time step the calculation of inner points in each region doesn't depends from each other. So the proposed method can be easily adopted for parallel execution.

For nonequilibrium calculations for solution of kinetic equations takes the main part of execution time. As it was shown in [4] it is possible to improve performance by redesign this part of program code according to vectorized computer ELECTRONIKASSBIS.

\section{Gas Cloud Expansion into Closed Vacuum Chamber}

The features of above technique are demonstrated on case of unsteady expansion of gas cloud of binary mixture of inert gas (argon) and condensing vapor (silver) into closed vacuum chamber filled by residual gas (air). Such flow is realized at shock tubes with chambers of high/low pressure, separated by membrane, at expansion of vapors generated by electrical explosion of thin wire in cylindrical vacuum tube, at expansion flow of substance evaporated by laser beam. The similar flows 6are considered in some astrophysical problems.

Due to high initial values of initial temperature and cooling rate the nonequilibrium homogeneous condensation can take place in such flow.

The initial cloud radii equals to $1 \mathrm{~mm}$, the chamber's radii equals to $5 \mathrm{~mm}$. The initial pressure and temperature of silver vapor equal to $5270 \mathrm{~Pa}$ and $2000 \mathrm{~K}$. The pressure and temperature of residual gas equal to $10 \mathrm{~Pa}$ and $300 \mathrm{~K}$, respectively. At Fig. 1 the r-t diagram for this flow is presented. One can see head shock wave, running at the low-pressure region, contact surface and centered rarefaction wave. Later head shock wave reflects from solid wall and, after interaction with contact surface, transforms into two shock waves moving in opposite directions. The left shock wave moves to the symmetry axis, reflects from it and comes back to solid wall. The right shock wave interacts with solid wall and this process repeats many times. The resulting flow has complex structure with lot of strong/weak discontinuities. As it is seen at Fig.1 that suggested numerical technique permits to receive "exact" picture of flow with all details.

The parameterized simulation of flows for various ratios $\mathrm{z}$ of chamber/cloud initial sizes and for various types of symmetry shows that for large $\mathrm{z}$ values the flow picture at initial times is simpler (less number of shock waves). The reason is that reflection of shocks from symmetry axis requires more time. Also there is difference of temperature behavior at solid wall. For $\mathrm{z}=1 / 5$ after increase of temperature at head shock wave it begins to decrease significantly. In case of $z=1 / 5$ the decreasing time is small and later temperature has stable level.

Against plane case in cylindrical/spherical flows the ending right characteristic of rarefaction wave transforms into shock wave as a result of interactions of characteristics of same family. The intensity of generated shock wave is greater in spherical case. 


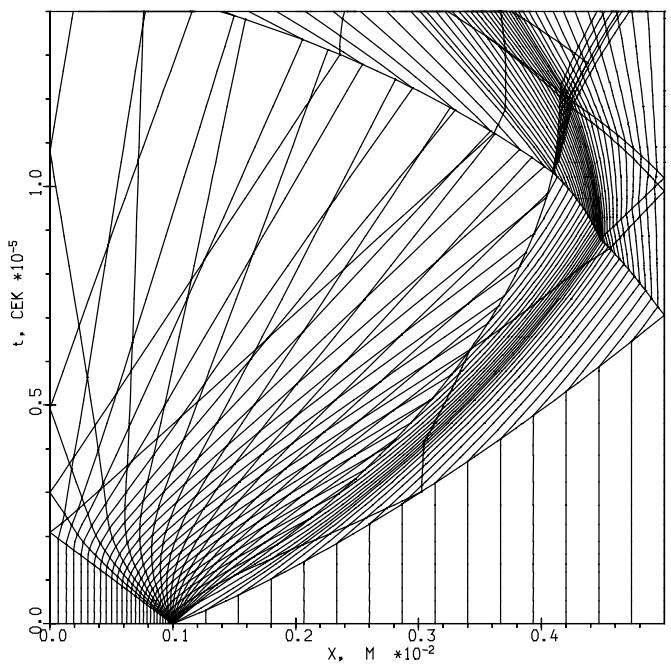

Fig. 1. The r-t diagram of unsteady expansion flow $(\mathrm{z}=1 / 5)$.

At Fig.2 the pressure-temperature diagram for first three trajectories behind contact surface is present. The results agree with usual pictures for nonequilibrium homogeneous condensation in high-speed flows. Initially the active changes of cluster size distribution function take place. But due to small values of cluster concentrations the mixture parameters vary according to adiabatic law. After formation of enough number of clusters (Wilson point) their fast growth step begins resulting in decreasing of super-saturation ratio. It is seen that maximal super-cooling value decreases with increasing of initial distance between trajectories and contact surface. That agrees with known data about nonequilibrium condensation.

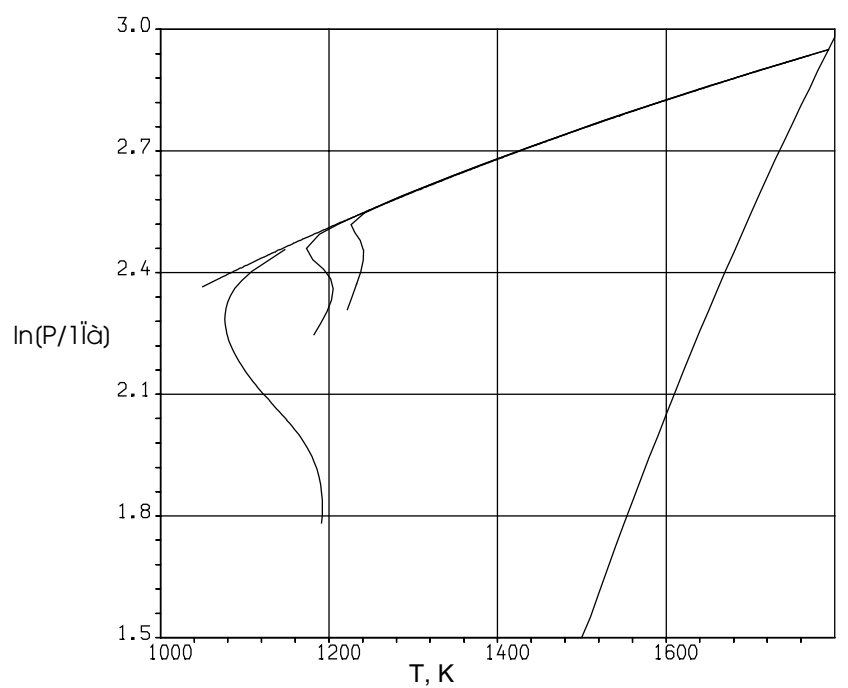

Fig. 2. The P-T diagram for unsteady expansion flow $(z=1 / 5)$. 


\section{Conclusion}

The suggested numerical technique can be used for wide class of 1D unsteady reacting flows with chock waves, detonation waves and other strong/weak discontinuities. The main advantages of above technique can be realized in case of reacting flows with unknown structure and without reliable kinetic mechanisms due to possibility to obtain all flow details.

\section{References}

1. Vinogradov A.V., Volkov V.A., Gidaspov V.Yu., Rozovski P.V. : Influence of Residual Gas on the Expansion of a Dence Gas Cloud in a Vacuum Chamber and itsInteraction with a Target or Wall. Technical Physics. 38 (1993) 946-948

2. Vinogradov A.V., Volkov V.A., Gidaspov V.Yu., Rozovski P.V. : Interaction of an Expanding Gas Cloud with a Perforated Screen. . Technical Physics. 42 (1997) 473-476

3. Volkov V.A., Muslaev A.V., Pirumov U.G., Rozovski P.V.: Nonequilibrium Condensation of Metal Vapor Mixed with Inert Gas in Nozzle Expansion in Cluster Beam Generator. Fluid Dynamics. 30 (1995) 335-486

4. Marasanov A.M., Rozovski P.V., Shebeko Yu.A. : Development of Software Package for Physical Gas Dynamics Problems for Vectorized Computers. Computer Technologies. 2 (1992) 223-231 (in Russian) 